\title{
Progress and prospects in stem cell therapy
}

\author{
Xiu-ling XU $\mathrm{U}^{1, \#, *}$, Fei $\mathrm{YI}^{2, \#}$, Hui-ze PAN ${ }^{1}$, Shun-lei DUAN ${ }^{1}$, Zhi-chao DING ${ }^{1}$, Guo-hong YUAN ${ }^{1}$, Jing $\mathrm{QU}^{1}$, Hai-chen ZHANG ${ }^{1}$, \\ Guang-hui LIU ${ }^{1, *}$ \\ ${ }^{1}$ National Laboratory of Biomacromolecules, Institute of Biophysics, Chinese Academy of Sciences, Beijing 100101, China; ${ }^{2}$ Depart- \\ ment of Molecular and Cellular Physiology, Stanford University School of Medicine, 265 Campus Drive, Stanford, CA 94305, USA
}

In the past few years, progress being made in stem cell studies has incontestably led to the hope of developing cell replacement based therapy for diseases deficient in effective treatment by conventional ways. The induced pluripotent stem cells (iPSCs) are of great interest of cell therapy research because of their unrestricted self-renewal and differentiation potentials. Proof of principle studies have successfully demonstrated that iPSCs technology would substantially benefit clinical studies in various areas, including neurological disorders, hematologic diseases, cardiac diseases, liver diseases and etc. On top of this, latest advances of gene editing technologies have vigorously endorsed the possibility of obtaining disease-free autologous cells from patient specific iPSCs. Here in this review, we summarize current progress of stem cell therapy research with special enthusiasm in iPSCs studies. In addition, we compare current gene editing technologies and discuss their potential implications in clinic application in the future.

Keywords: induced pluripotent stem cells (iPSCs); stem cell therapy; gene editing; neurological disorders; hematologic diseases; cardiac diseases; liver diseases

Acta Pharmacologica Sinica (2013) 34: 741-746; doi: 10.1038/aps.2013.77

\section{Introduction}

Stem cells are special cells which are able to differentiate into few (for multipotent adult stem cells) or any (for pluripotent embryonic stem cells, PSCs) lineage-specific cell types while self-renewing extensively to generate more stem cells. Because of their potency of differentiation, stem cells are often foreseen as the hope of novel cell replacement based therapies to regenerate tissues/organs damaged by injury or disease. Transplantation of stem cells or their derivatives into respective tissues or organs is considered as one of the most promising remedies for many incurable diseases in traditional meanings, of which bone marrow transplantation for treating leukemia is the best-known example ${ }^{[1]}$. Unfortunately, immune compatible cells are hardly obtainable for any given patient, because of the specificity and complexity of human immune system. In this regard, human induced pluripotent stem cells (hiPSCs) and gene editing technologies are believed to offer an unprecedented solution for obtaining sufficient healthy autologous cells. Here we review recent important advances of

\footnotetext{
"These authors contributed equally to this paper.

* To whom correspondence should be addressed.

E-mail ghliu@ibp.ac.cn (Guang-hui LIU); xiulingxu@hotmail.com (Xiu-ling XU)

Received 2013-03-19 Accepted 2013-05-16
}

stem cell based research on neurodegenerative diseases, blood diseases, cardiac diseases, and liver related diseases, with special emphasis on the human ESCs (hESCs) and hiPSCs based research with clinical intention.

\section{Stem cell based therapies for neurological disorders}

Neurological disorders caused by dysfunctions of neurons or glial cells, such as Parkinson's disease (PD), amyotrophic lateral sclerosis (ALS), Huntington's disease (HD) and strokes, are often devastating and life-threatening ${ }^{[2,3]}$. Though currently no cure is available for these diseases, hPSCs based therapies are proposed to provide a symptomatic relief, therefore representing a promising option for developing new treatment.

PD is caused by the selective death of dopaminergic (DA) neurons in the substantia nigra of midbrain. Replacing impaired DA neurons with new cells is one of the straightforward strategies to treat this disease. Trails using fetal ventral mesencephalic tissue which is enriched of neural stem cells (NSCs) for PD treatment began decades ago ${ }^{[4]}$, and data showed that the transplanted NSCs could engraft and survive for years. Encouragingly, unlike patient's own neurons, the engrafted neurons did not contain a-synuclein aggregates, an important pathological hallmark of $\mathrm{PD}^{[5]}$. However, due to ethical conflicts and limitations of fetal NSCs, new sources 
of NSCs for transplantation need to be explored. Because of the unlimited self-renewal and differentiation capability, hESCs and PD patient hiPSCs are considered promising candidate resources for treating PD. As a result, many groups have sought to establish protocols to efficiently differentiate hESCs/hiPSCs into DA neurons ${ }^{[6,7]}$. It has been reported that transplantation of these hESCs/hiPSCs derived DA neurons into a rodent model of PD can substantially reverse animal behavioral dysfunction, suggesting a clinical potential of this treatment ${ }^{[8-11]}$. Moreover, Wernig and colleagues found that 6-hydroxydopamine (6-OHDA) rodent model of PD showed behavioral improvement after the engraftment of hiPSCs derived DA neurons, which strongly advocates the concept of hiPSCs mediated autologous cell therapy. On the other hand, the recent advances in gene correction technologies using zinc-finger nucleases (ZFN), transcription activator-like effector nucleases (TALEN), clustered regularly interspaced short palindromic repeats (CRISPR)/CRISPR-associated systems $(\mathrm{Cas})^{[12,13]}$, or helper-dependent adenoviral vector (HDAdV) have further expanded the versatility of using patient hiPSCs to treat neurologic diseases for a less risk of immune rejection ${ }^{[14-18]}$. These studies suggested that genetic defects could be effectively corrected before applying stem cell based therapy for PD or other neurological diseases.

\section{Stem cell based therapies for the hematologic diseases}

Hematologic diseases include a wide range of the acute and chronic alternations in the hematological cell lineages. Among them, leukemia, anemia, multiple myeloma, lymphomas, and melanoma are common examples, many of which are lifethreatening. Since the first bone marrow transplantation performed in 1950s, hematopoietic stem cells (HSCs) transplantation has been proved as the most successful application of stem cell therapy in the clinic. Compared to conventional chemotherapy, stem cell based therapy presents a more effective and less toxic way of treating these diseases. When succeeded, HSCs transplantation could be curative for all sorts of genetic blood disorders like thalassemia and immune deficiency, as well as malignancies like leukemia and lymphoma. Till now, HSCs transplantation remains the only curative therapy for certain genetic diseases of the hematopoietic system. Cord blood, bone marrow and peripheral blood (after immortalization) are the main sources of obtaining HSCs. No significant difference was observed in patients survived after transplantation with HSCs from different origins. Unfortunately, the application and efficacy of HSC transplantation are limited by the shortage of suitable donors and inefficient in vitro expansion of HSCs. Moreover, low efficiency of gene delivery/correction in HSCs and safety concerns due to random viral integration further restrict its application. Recent advancement in hiPSCs may open a new era for curing such severe hematopoietic diseases. Owing to their remarkable capacity of differentiation upon appropriate stimuli, hESCs and hiPSCs exhibit fantastic potential in cell replacement therapy for hematologic diseases. Recently, direct differentiation protocols have been developed and improved for efficient generation of HSCs from
hESCs and hiPSCs. The derived HSCs are capable of further differentiation into specific mature blood components including erythroid cells, platelets, dendrite cells, natural killer cells, and $T$ cells etc ${ }^{[19]}$.

For treating the non-inherited blood disorders such as acquired aplastic anemia, hiPSCs derived HSCs are suitable for autologous transplantation without any genetic manipulation since no pathological mutation is present. For the inherited blood diseases such as thalassemia and sickle cell anemia, gene correction is mandatory to repair pathological mutations in patient hiPSCs. For instance, most of the gene correction works done so far were in hiPSCs from patients with hemoglobinopathy. The disease related mutations were corrected either by in situ gene repair through homologous recombination or by transgenic introduction of healthy gene at a safe harbor locus ${ }^{[18,20-22]}$. In both manners, it has been shown that disease symptoms were rescued at cellular levels. However, due to the difficulties of grafting of human HSCs into the host animals, more efforts should be put in continuous studies to further ensure if disease defects are functionally rescued in animal models.

\section{Stem cell based therapy in cardiac diseases}

Heart failure is a common pathological condition which results from different reasons including myocardial infarction and chronic cardiac disorders. Cardiomyocytes in adult mammals have very limited innate ability to regenerate, which makes it extremely difficult for the heart to recover after injury. Limitation of conventional treatments, such as interventions and cardiac transplantation, has been demonstrated clearly. On the other hand, the stem cell therapy of heart diseases has received enormous attention with the hope of rescuing patients with heart failure. Standing out as a promising way for cardiac repair, regeneration of functional cardiomyocytes is highly demanded for treating heart failure.

In principle, cardiomyocytes can be generated by two different ways, differentiation from hPSCs or trans-differentiation from other somatic lineages. As a classic way, spontaneous cardiomyocytes differentiation from hPSCs through embryoid body (EB) formation in vitro is very inefficient ( 1\%-10\% cardiomyocytes produced). It has been found that many factors would facilitate a more specific and efficient differentiation, which may lead to the establishment of a refined protocol applicable for the purpose of regenerative medicine. Many cardiac lineage inducing cytokines and signaling modulating small compounds discovered from developmental studies have been widely tested in the in vitro culture system. Among them, activin A and BMP4 have been shown to effectively drive the differentiation of hESCs towards cardiomyocytes with higher efficiency (up to $30 \%)^{[23]}$. The derived cardiomyocytes were not only highly resembling their primary counterparts in terms of morphological features and gene expression patterns, but also capable of partially rescuing heart failure in infarcted rat hearts after transplantation. Combined with activin A and BMP4, a new report has shown that bFGF could efficiently induce hESCs into primitive streak-like cells 
and cardiac mesoderm ${ }^{[24]}$. This protocol subsequently used canonical Wnt inhibitor DKK1 and VEGF to promote cardiomyocyte differentiation and further improved the efficiency of differentiation to around $50 \%$. In addition, small molecules have also been shown to play important roles in establishing a reliable and economical approach for cardiac regeneration. Recently, Lian et al reported that both hESCs and hiPSCs could be induced to more compatible cardiomyocytes through temporal modulation of Wnt signaling using small compound ${ }^{[25]}$. By administration of GSK3-specific inhibitor on the first day of differentiation and Wnt inhibitor IWP2 or IWP4 on the third day, the purity of differentiated cardiomyocytes was dramatically increased (higher than $82 \%$ ). Meanwhile, some small molecules are suggested to have biphasic effects on cardiomyocyte differentiation, like ITD-1 being recently reported by Willems et $a l^{[26]}$. Administration of this compound at the early stage of differentiation tended to induce mesoderm formation while its later effect specifically promoted cardiomyocyte differentiation by inhibiting formation of other lineages. Besides small molecules, it has been reported that extracellular matrix components, such as Matrigel, may also contribute to the differentiation of cardiomyocytes. In addition to the conventional inductive signals activin A, BMP4, and bFGF, Zhang et al found in a recent study that, by using Matrigel to make a matrix sandwich with monolayer of hPSCs in the middle, functional cardiomyocytes with an extreme high purity (up to $98 \%$ ) can be obtained ${ }^{[27]}$. Besides differentiation, 3D cell technology can also be used to facilitate transplantation. Assisted by tissue engineering, Kawamura et al reported a 3D reconstruction methodology dramatically improved the efficiency of engraftment after cardiomyocyte transplantation ${ }^{[28]}$ (Table 1).

Compared to the improved differentiation methods, another exciting and inspiring breakthrough in the field is the successful conversion of cardiomyocytes from other lineage committed cells, to bypass all safety risks associated with the pluripotency. For example, Qian et al demonstrated both functional evidence and decreased disease symptom on cardiomyocytes transdifferentiated from cardiac fibroblasts of infarcted mouse heart ${ }^{[29]}$. Similarly, Song et al reported a successful directed lineage reprogramming of functional cardiomyocytes from non-myocytes of injured mouse heart ${ }^{[30]}$, suggesting a novel strategy for the cardiac repair in vivo. In summary, though big progress has been made in the field, it is still a long way to go in order to achieve the goal of healing or curing heart diseases in the future.

\section{Hepatic differentiation for cell-based therapy}

End-stage liver diseases (ESLD), such as hepatic cirrhosis, viral hepatitis and hepatocarcinoma may lead to severe hepatic failure which is irreversible. Currently, orthotropic liver transplantation (OLT) is the only effective treatment for ESLD. However, the lack of donor liver and immune-compatibility problems are two major obstacles to the routine clinical practice of OLT. Human PSCs, including hESCs and hiPSCs represent a promising alternative of cell therapy for liver diseases. Recently, a lot of studies have demonstrated that hESCs can be efficiently differentiated into functional hepatocytes both in vitro and in vivo ${ }^{[31-33]}$. Unfortunately, their clinical application is restricted by the immunological and ethical issues. Now, those problems can be avoided by generating hiPSCs directly from patient. hiPSCs have been successfully produced from somatic cells through cell reprogramming and differentiated into functional hepatocytes ${ }^{[34,35]}$. Although the clinical potential of hiPSCs derived hepatocytes is enormous, the long-term functionality, effectiveness and safety issues after cell transplantation will still need intensive investigation.

In short, the strategy of hPSC-based cell replacement therapy is summarized as Figure 1.

\section{Gene editing and stem cell therapy}

Gene editing technology in hPSCs provides a powerful tool for treating many genetic diseases. However, for quite a long time the extremely low efficiency of gene targeting in hPSCs through conventional homologous recombinationbased method hindered its application. Several powerful gene targeting tools, including ZFN, TALEN, CRISPR/Cas, and HDAdV, have been successfully developed. These new technologies have tremendously elevated the targeting efficiency and been widely used in the targeted correction of disease-specific mutations, as well as gene knock-in studies ${ }^{[14,15,18,21,36-39]}$. By using these novel gene editing tools, several groups have made great progress in the correction of pathogenic mutations in patient-derived hiPSCs ${ }^{[14,21,39-47]}$, which implies an encouraging perspective of this technology in the future.

In 2011, Jaenisch and colleagues successfully corrected an a-Synuclein mutation using ZFN in hiPSCs derived from patients with Parkinson's disease ${ }^{[14]}$. In another study, researchers reported correction of mutated $a_{1}$-ANTITR YPSIN gene without any "footprint" in the genome by ZFN as well as piggyBAC transposon ${ }^{[45]}$. Though ZFN-mediated gene editing has a relatively high efficiency, it faces a major concern of

Table 1. Summary of cardiomyocytes directed differentiation of hPSCs.

\begin{tabular}{lllll}
\hline \multicolumn{1}{c}{ Cell line } & Terminal cells & Additional factors & Efficiency & Author \\
\hline H7 human ESCs & Cardiomyocytes & Activin A, BMP4 & $>30 \%$ & Laflamme et al 2007 $^{[23]}$ \\
Human ESCs (H1, HES2) & Cardiomyocytes & BMP4, activin A, bFGF, VEGF, DKK1, & $\sim 50 \%$ & Yang et al 2008 \\
Human ESCs (H9, H13, H14); hiPSCs & Cardiomyocytes & B-catenin shRNA, activin A, BMP4, CHIR99021, or IWP2 & $>82 \%$ & Lian et al 2012 $^{[25]}$ \\
Human ESCs (H9) & Cardiomyocytes & BMP4, activin A, bFGF, VEGF, DKK1, ITD-1 & $\sim 60 \%$ & Willems et al 2012 \\
Human ESCs (H1, H9); hiPSCs & Cardiomyocytes & Activin A, BMP4, bFGF & $\sim 98 \%$ & Zhang et al 2012 \\
& & & & \\
\hline
\end{tabular}




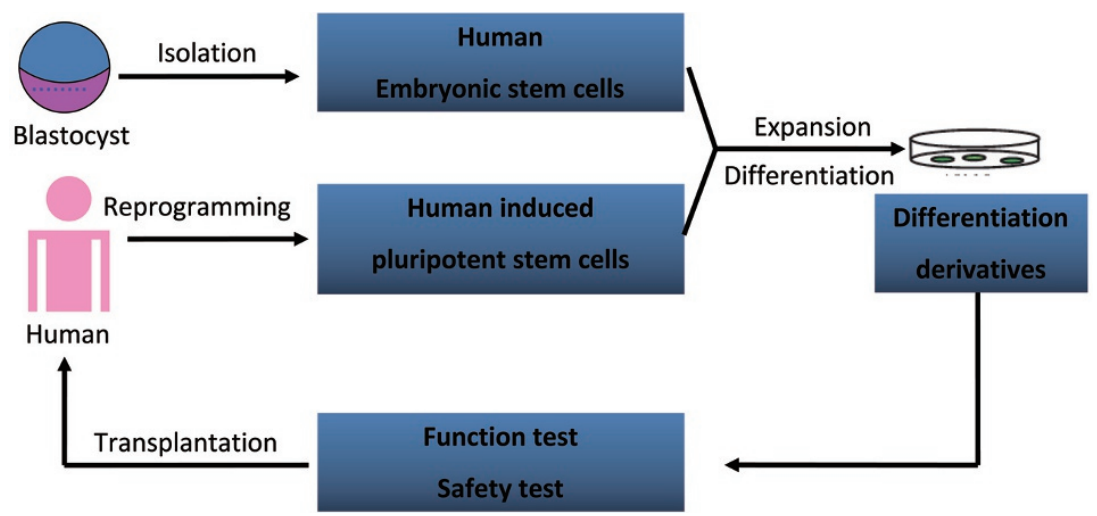

Figure 1. Potential strategy of human PSC-based therapy for human diseases.

potential off-target effects. Furthermore, ZFN may also cause genetic toxicity ${ }^{[48,49]}$. Recently, TALEN has been developed as a novel gene editing technique in hiPSCs ${ }^{[50]}$. Compared to ZFN, engineered TALEN possesses similar or higher gene targeting efficiency ${ }^{[50]}$. However, similarly to ZFN, TALEN also functions as a nuclease. Therefore, its potential off-target effects will still need to be comprehensively examined before the translation into clinic ${ }^{[4]}$.

A novel HDAdV-mediated gene targeting method has recently been demonstrated as an efficient and safe strategy for correcting gene mutations ${ }^{[15,17,18,51]}$. HDAdV possesses a relatviely high gene targeting efficiency, compared to other parallel methods. More importantly, since the HDAdV overcomes the potential toxicity commonly associated with viral vector, it has been proved to be a safe method for gene correction in patient hiPSCs. Applying the HDAdV strategy, Liu et al reported successful correction of LMNA gene mutations in hiPSCs derived from patients of progeria ${ }^{[1,52]}$.

Recently, three laboratories independently reported the success of correcting mutations on $\beta$-Globin $(H B B)$ gene in sickle cell patient hiPSCs by different gene targeting strategies ${ }^{[18,39,47]}$. In two studies, the authors employed ZFN as the gene editing tool and obtained targeted cell lines with precise correction of $H B B^{[39,47]}$. The off-target effects in whole genome are still required to be examined for safety concern. Meanwhile, $\mathrm{Li}$ et al utilized a HDAdV-mediated gene targeting strategy to correct the $H B B$ mutation. In addition to the high efficiency of gene correction, a single HDAdV correction vector was competent to correct all implicated mutations at the $H B B$ locus, as the full HBB genomic sequence was included in the vector ${ }^{[18]}$.

In conclusion, targeted gene correction of pathological mutations in patient hiPSCs provides a promise for regenerative medicine (Figure 2). However, it is essential to ensure that the gene editing procedures do not introduce any unexpected mutation. To this end, more efficient and safe gene correction strategies, as well as more robust whole genome sequencing tools, may be needed for the generation of mutation-free hiPSCs before the therapeutic application in the future.

Cardiovascular diseases, neurodegenerative disorders and liver diseases are leading causes of death and paralyses worldwide. Though still at an early stage, current progress of stem cell research in these therapeutic areas is driving stem cell based therapy towards practice. As many ones believe in, stem cell based therapy is not an unrealistic goal in the near

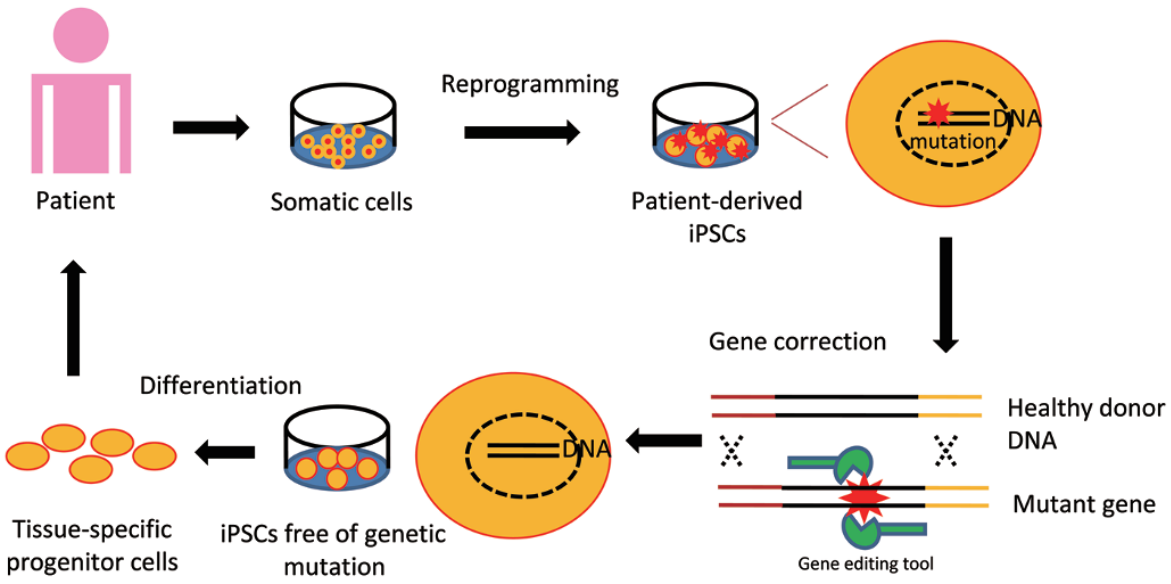

Figure 2. Mutant genes in patient-derived iPSCs are corrected by the use of gene editing tools (eg ZFN, TALEN, CRISPR/Cas, HDAdV). The differentiation derivatives from corrected patient iPSCs hold the potential to be employed in autologous cell therapy. 
future if some essential questions are resolved definitively. The safety and scaling issues are still hindering its application into clinic. Taken transplantation into consideration, there are more issues requiring special attention. Targeted gene correction technologies should be optimized to remove all pathogenic mutations without affecting genomic integrity. The immune rejection related problems, although controversial, represent another big obstacle. The structural and functional integration of transplanted cells into the host tissue is also important to minimize the damage of the procedure. At the end, pathological improvement in long run must be carefully evaluated to ensure the efficacy of the treatment, which requires a prolonged survival of engrafted cells. Once all technical and ethical barriers are being removed, we will be able to eventually benefit enormously from what we are devastatingly learning and fighting for the success of stem cell based therapy in the future.

\section{Acknowledgements}

Guang-hui LIU is supported by the Strategic Priority Research Program of the Chinese Academy of Sciences (XDA01020312), the National Natural Science Foundation of China (NSFC) (81271266, 31222039), the Thousand Young Talents program of China, the National Laboratory of Biomacromolecules (013kf05, 2013kf11), and State Key Laboratory of Drug Research (SIMM1302KF-17). Xiu-ling XU is supported by NSFC (31201111).

\section{References}

1 Kebriaei P, Poon LM. The role of allogeneic hematopoietic stem cell transplantation in the therapy of patients with acute lymphoblastic leukemia. Curr Hematol Malig Rep 2012; 7: 144-52.

2 Ilieva H, Polymenidou M, Cleveland DW. Non-cell autonomous toxicity in neurodegenerative disorders: ALS and beyond. J Cell Biol 2009; 187: 761-72.

3 Ross CA, Poirier MA. Protein aggregation and neurodegenerative disease. Nat Med 2004; 10 : S10-7.

4 Lindvall O, Widner H, Rehncrona S, Brundin P, Odin P, Gustavii B, et al. Transplantation of fetal dopamine neurons in Parkinson's disease: one-year clinical and neurophysiological observations in two patients with putaminal implants. Ann Neurol 1992; 31: 155-65.

5 Mendez I, Viñuela A, Astradsson A, Mukhida K, Hallett P, Robertson $\mathrm{H}$, et al. Dopamine neurons implanted into people with Parkinson's disease survive without pathology for 14 years. Nat Med 2008; 14 : 507-9.

$6 \mathrm{Kim} \mathrm{JH}$, Auerbach JM, Rodríguez-Gómez JA, Velasco I, Gavin D, Lumelsky $\mathrm{N}$, et al. Dopamine neurons derived from embryonic stem cells function in an animal model of Parkinson's disease. Nature 2002; 418: 50-6.

7 Perrier AL, Tabar V, Barberi T, Rubio ME, Bruses J, Topf N, et al. Derivation of midbrain dopamine neurons from human embryonic stem cells. Proc Natl Acad Sci U S A 2004; 101: 12543-8.

8 Wernig M, Zhao JP, Pruszak J, Hedlund E, Fu D, Soldner F, et al. Neurons derived from reprogrammed fibroblasts functionally integrate into the fetal brain and improve symptoms of rats with Parkinson's disease. Proc Natl Acad Sci U S A 2008; 105: 5856-61.

9 Zhang W, Duan S, Li Y, Xu X, Qu J, Zhang W, et al. Converted neural cells: induced to a cure? Protein Cell 2012; 3: 91-7.
10 Caiazzo M, Dell'Anno MT, Dvoretskova E, Lazarevic D, Taverna S, Leo $D$, et al. Direct generation of functional dopaminergic neurons from mouse and human fibroblasts. Nature 2011; 476: 224-7.

11 Kim J, Su SC, Wang H, Cheng AW, Cassady JP, Lodato MA, et al. Functional integration of dopaminergic neurons directly converted from mouse fibroblasts. Cell Stem Cell 2011; 9: 413-9.

12 Mali P, Yang L, Esvelt KM, Aach J, Guell M, DiCarlo JE, et al. RNAguided human genome engineering via Cas9. Science 2013; 339: 823-6.

13 Wang $\mathrm{H}$, Yang $\mathrm{H}$, Shivalila CS, Dawlaty MM, Cheng AW, Zhang F, et al. One-step generation of mice carrying mutations in multiple genes by CRISPR/Cas-mediated genome engineering. Cell 2013; 153: 910-8.

14 Soldner F, Laganière J, Cheng AW, Hockemeyer D, Gao Q, Alagappan $\mathrm{R}$, et al. Generation of isogenic pluripotent stem cells differing exclusively at two early onset Parkinson point mutations. Cell 2011; 146: 318-31.

15 Liu GH, Suzuki K, Qu J, Sancho-Martinez I, Yi F, Li M, et al. Targeted gene correction of laminopathy-associated LMNA mutations in patient-specific iPSCs. Cell Stem Cell 2011; 8: 688-94.

16 Hockemeyer D, Wang H, Kiani S, Lai CS, Gao Q, Cassady JP, et al. Genetic engineering of human pluripotent cells using TALE nucleases. Nat Biotechnol 2011; 29: 731-4.

17 Liu GH, Qu J, Suzuki K, Nivet E, Li M, Montserrat N, et al. Progressive degeneration of human neural stem cells caused by pathogenic LRRK2. Nature 2012; 491: 603-7.

18 Li M, Suzuki K, Qu J, Saini P, Dubova I, Yi F, et al. Efficient correction of hemoglobinopathy-causing mutations by homologous recombination in integration-free patient iPSCs. Cell Res 2011; 21: 1740-4.

19 Kimbrel EA, Lu SJ. Potential clinical applications for human pluripotent stem cell-derived blood components. Stem Cells Int 2011; 2011: 273076.

20 Chang CJ, Bouhassira EE. Zinc-finger nuclease-mediated correction of alpha-thalassemia in iPS cells. Blood 2012; 120: 3906-14.

21 Zou J, Mali P, Huang X, Dowey SN, Cheng L. Site-specific gene correction of a point mutation in human iPS cells derived from an adult patient with sickle cell disease. Blood 2011; 118: 4599-608.

22 Wang Y, Zheng CG, Jiang Y, Zhang J, Chen J, Yao C, et al. Genetic correction of beta-thalassemia patient-specific iPS cells and its use in improving hemoglobin production in irradiated SCID mice. Cell Res 2012; 22: 637-48.

23 Laflamme MA, Chen KY, Naumova AV, Muskheli V, Fugate JA, Dupras $\mathrm{SK}$, et al. Cardiomyocytes derived from human embryonic stem cells in pro-survival factors enhance function of infarcted rat hearts. Nat Biotechnol 2007; 25: 1015-24.

24 Yang L, Soonpaa MH, Adler ED, Roepke TK, Kattman SJ, Kennedy M, et al. Human cardiovascular progenitor cells develop from a $\mathrm{KDR}^{+}$ embryonic-stem-cell-derived population. Nature 2008; 453: 524-8.

25 Lian X, Hsiao C, Wilson G, Zhu K, Hazeltine LB, Azarin SM, et al. Robust cardiomyocyte differentiation from human pluripotent stem cells via temporal modulation of canonical Wnt signaling. Proc Natl Acad Sci U S A 2012; 109: E1848-57.

26 Willems E, Cabral-Teixeira J, Schade D, Cai W, Reeves P, Bushway PJ, et al. Small molecule-mediated TGF-beta type II receptor degradation promotes cardiomyogenesis in embryonic stem cells. Cell Stem Cell 2012; 11: 242-52.

27 Zhang J, Klos M, Wilson GF, Herman AM, Lian X, Raval KK, et al. Extracellular matrix promotes highly efficient cardiac differentiation of human pluripotent stem cells: the matrix sandwich method. Circ Res 2012; 111: 1125-36.

28 Kawamura M, Miyagawa S, Miki K, Saito A, Fukushima S, Higuchi T, et al. Feasibility, safety, and therapeutic efficacy of human induced 
pluripotent stem cell-derived cardiomyocyte sheets in a porcine ischemic cardiomyopathy model. Circulation 2012; 126: S29-37.

29 Qian L, Huang Y, Spencer Cl, Foley A, Vedantham V, Liu L, et al. In vivo reprogramming of murine cardiac fibroblasts into induced cardiomyocytes. Nature 2012; 485: 593-8.

30 Song K, Nam YJ, Luo X, Qi X, Tan W, Huang GN, et al. Heart repair by reprogramming non-myocytes with cardiac transcription factors. Nature 2012; 485: 599-604.

31 Agarwal S, Holton KL, Lanza R. Efficient differentiation of functional hepatocytes from human embryonic stem cells. Stem Cells 2008; 26 : 1117-27.

32 Duan Y, Ma X, Zou W, Wang C, Bahbahan IS, Ahuja TP, et al. Differentiation and characterization of metabolically functioning hepatocytes from human embryonic stem cells. Stem Cells 2010; 28 : 674-86.

33 Yi F, Qu J, Li M, Suzuki K, Kim NY, Liu GH, et al. Establishment of hepatic and neural differentiation platforms of Wilson's disease specific induced pluripotent stem cells. Protein Cell 2012; 3: 855-63.

34 Chen YF, Tseng CY, Wang HW, Kuo HC, Yang VW, Lee OK. Rapid generation of mature hepatocyte-like cells from human induced pluripotent stem cells by an efficient three-step protocol. Hepatology 2012; 55: 1193-203.

35 Kajiwara M, Aoi T, Okita K, Takahashi R, Inoue H, Takayama N, et al. Donor-dependent variations in hepatic differentiation from humaninduced pluripotent stem cells. Proc Natl Acad Sci U S A 2012; 109: 12538-43.

36 Howden SE, Gore A, Li Z, Fung HL, Nisler BS, Nie J, et al. Genetic correction and analysis of induced pluripotent stem cells from a patient with gyrate atrophy. Proc Natl Acad Sci U S A 2011; 108 : 6537-42.

37 Papapetrou EP, Lee G, Malani N, Setty M, Riviere I, Tirunagari LM, et al. Genomic safe harbors permit high beta-globin transgene expression in thalassemia induced pluripotent stem cells. Nat Biotechnol 2011; 29: 73-8.

38 Raya A, Rodríguez-Pizà I, Guenechea G, Vassena R, Navarro S, Barrero $\mathrm{MJ}$, et al. Disease-corrected haematopoietic progenitors from Fanconi anaemia induced pluripotent stem cells. Nature 2009; 460: 53-9.

39 Sebastiano V, Maeder ML, Angstman JF, Haddad B, Khayter C, Yeo DT, et al. In situ genetic correction of the sickle cell anemia mutation in human induced pluripotent stem cells using engineered zinc finger nucleases. Stem Cells 2011; 29: 1717-26.

40 Asuri P, Bartel MA, Vazin T, Jang JH, Wong TB, Schaffer DV. Directed evolution of adeno-associated virus for enhanced gene delivery and gene targeting in human pluripotent stem cells. Mol Ther 2012; 20 : 329-38.

41 DeKelver RC, Choi VM, Moehle EA, Paschon DE, Hockemeyer D, Meijsing $\mathrm{SH}$, et al. Functional genomics, proteomics, and regulatory DNA analysis in isogenic settings using zinc finger nuclease-driven transgenesis into a safe harbor locus in the human genome. Genome
Res 2010; 20: 1133-42.

42 Hockemeyer D, Soldner F, Beard C, Gao Q, Mitalipova M, DeKelver RC, et al. Efficient targeting of expressed and silent genes in human ESCs and iPSCs using zinc-finger nucleases. Nat Biotechnol 2009; 27: 851-7.

43 Lombardo A, Cesana D, Genovese P, Di Stefano B, Provasi E, Colombo $\mathrm{DF}$, et al. Site-specific integration and tailoring of cassette design for sustainable gene transfer. Nat Methods 2011; 8: 861-9.

44 Mussolino C, Morbitzer R, Lütge F, Dannemann N, Lahaye T, Cathomen T. A novel TALE nuclease scaffold enables high genome editing activity in combination with low toxicity. Nucleic Acids Res 2011; 39: 9283-93.

45 Yusa K, Rashid ST, Strick-Marchand H, Varela I, Liu PQ, Paschon DE, et al. Targeted gene correction of alpha1-antitrypsin deficiency in induced pluripotent stem cells. Nature 2011; 478: 391-4.

46 Zou J, Maeder ML, Mali P, Pruett-Miller SM, Thibodeau-Beganny S, Chou BK, et al. Gene targeting of a disease-related gene in human induced pluripotent stem and embryonic stem cells. Cell Stem Cell 2009; 5: 97-110.

47 Zou J, Sweeney CL, Chou BK, Choi U, Pan J, Wang H, et al. Oxidasedeficient neutrophils from $\mathrm{X}$-linked chronic granulomatous disease iPS cells: functional correction by zinc finger nuclease-mediated safe harbor targeting. Blood 2011; 117: 5561-72.

48 Gabriel R, Lombardo A, Arens A, Miller JC, Genovese P, Kaeppel C, et al. An unbiased genome-wide analysis of zinc-finger nuclease specificity. Nat Biotechnol 2011; 29: 816-23.

49 Pattanayak V, Ramirez CL, Joung JK, Liu DR. Revealing off-target cleavage specificities of zinc-finger nucleases by in vitro selection. Nat Methods 2011; 8: 765-70.

50 Miller JC, Tan S, Qiao G, Barlow KA, Wang J, Xia DF, et al. A TALE nuclease architecture for efficient genome editing. Nat Biotechnol 2011; 29: 143-8.

51 Suzuki K, Mitsui K, Aizawa E, Hasegawa K, Kawase E, Yamagishi T, et al. Highly efficient transient gene expression and gene targeting in primate embryonic stem cells with helper-dependent adenoviral vectors. Proc Natl Acad Sci U S A 2008; 105: 13781-6.

52 Liu GH, Barkho BZ, Ruiz S, Diep D, Qu J, Yang SL, et al. Recapitulation of premature ageing with iPSCs from Hutchinson-Gilford progeria syndrome. Nature 2011; 472: 221-5.

This work is licensed under the Creative Commons Attribution-NonCommercial-No Derivative Works 3.0 Unported License. To view a copy of this license, visit http://creativecommons.org/licenses/ by-nc-nd/3.0/ 